\title{
Characterization of the Human Exposome by a Comprehensive and Quantitative Large-Scale Multianalyte Metabolomics Platform
}

\author{
Raúl González-Domínguez,* Olga Jáuregui, María Isabel Queipo-Ortuño, and Cristina Andrés-Lacueva*
}

Cite This: Anal. Chem. 2020, 92, 13767-13775

Read Online

ABSTRACT: The exposome, defined as the cumulative measure of external exposures and associated biological responses throughout the lifespan, has emerged in recent years as a cornerstone in biomedical sciences. Metabolomics stands out here as one of the most powerful tools for investigating the interplay between the genetic background, exogenous, and endogenous factors within human health. However, to address the complexity of the exposome, novel methods are needed to characterize the human metabolome. In this work, we have optimized and validated a multianalyte metabolomics platform for large-scale quantitative exposome research in plasma and urine samples, based on the use of simple extraction methods and high-throughput metabolomic fingerprinting. The methodology enables, for the first time, the simultaneous characterization of the endogenous metabolome, foodrelated metabolites, pharmaceuticals, household chemicals, environmental pollutants, and microbiota derivatives, comprising more than 1000 metabolites in total. This comprehensive and quantitative investigation of the exposome is achieved in short run times, through simple extraction methods requiring small-sample volumes, and using integrated quality control procedures for ensuring data quality. This metabolomics approach was satisfactorily validated in terms of linearity, recovery, matrix effects, specificity, limits of quantification, intraday and interday precision, and carryover. Furthermore, the clinical potential of the methodology was demonstrated in a dietary intervention trial as a case study. In summary, this study describes the optimization, validation, and application of a multimetabolite platform for comprehensive and quantitative metabolomics-based exposome research with great utility in large-scale epidemiological studies.

$\mathrm{O}$ ver the past few years, increasing evidence has demonstrated that environmental factors have more impact on health status than originally thought, beyond the well-recognized role of the intrinsic genome. ${ }^{1}$ The "exposome" has emerged here as a novel research paradigm in biomedical sciences that could be defined as the totality of external exposures (e.g., diet, medication, pollution) and associated biological responses (i.e., endogenous processes) throughout the lifespan. ${ }^{2,3}$ Considering this complex interplay among multiple biological and lifestyle conditions, metabolomics is currently one of the most powerful technologies for holistically deciphering the molecular mechanisms driving the final phenotype, given that metabolites are direct indicators of the functional status of biological systems. In recent years, the use of metabolomics has exponentially increased in nutritional, pharmacological, and toxicological research., ${ }^{4,5}$ Other lifestyle habits with deep impact on the human metabolome and health status include smoking, the consumption of alcohol and illicit drugs, and physical activity. ${ }^{6,7}$ Within this tangled metabolic framework, the prominent role of gut microbiota in host health should also be noted, since it is involved in the absorption of nutrients, the metabolism of xenobiotics, the synthesis of biologically active metabolites, and other functions.
The vast chemical complexity and dynamic range of the exposome-being endogenous and food-derived metabolites normally found in the picomolar to millimolar ( $\mathrm{pM}-\mathrm{mM}$ ) concentration range, while pollutants can be present at levels 3 orders of magnitude lower ${ }^{10}$ - make the application of highthroughput and versatile techniques mandatory. Traditional approaches based on targeted biomonitoring of specific biomarkers are inherently biased to the predefined set of analytes, usually being time-consuming and requiring large sample volumes. ${ }^{11}$ On the other hand, nontargeted metabolomics allows the coverage to be expanded to thousands of metabolites, considering not only exogenous compounds but also endogenous metabolites altered by the exposure, thereby providing a simultaneous overview on both external factors and the associated phenotypic variations. ${ }^{12,13}$ Nonetheless, non-

Received: May 10, 2020

Accepted: September 23, 2020

Published: September 23, 2020 
targeted approaches also present critical limitations: (i) lower sensitivity than targeted methods, which impedes the detection of minor metabolites; (ii) lower reproducibility due to the semiquantitative nature of the measurement; and (iii) the limited number of compounds characterized in metabolomics databases, which hinders their annotation and subsequent biological interpretation. ${ }^{11,14}$ Accordingly, in recent years, more and more authors have emphasized the need to move to large-scale quantitative metabolomics for comprehensive exposome research, which would enable cross-cohort and interlaboratory comparisons, the pooling of data, and defining "quantitative reference metabolomes". ${ }^{15-17}$

The aim of the present work was to accomplish, for the first time, the development of a high-throughput metabolomics method to capture the metabolic complexity of the human exposome in a comprehensive and quantitative fashion. To this end, we considered endogenous metabolites involved in central pathways, food-related metabolites, commonly consumed drugs, chemicals coming from personal care products, plastic additives and environmental pollution, biomarkers of other lifestyle habits (e.g., smoking, alcohol consumption), and metabolites related to microbiota detoxification processes, food metabolism, and host health. To facilitate its implementation in large-scale epidemiological studies, we optimized simple, efficient, and small-sample consuming extraction methods for both urine and plasma samples, combined with a highthroughput metabolomics platform for comprehensive and quantitative characterization of the exposome in short run times. This metabolomics approach was subsequently applied to samples collected in a long-term intervention trial with olive oil as a case study to perform a clinical validation of the methodology.

\section{EXPERIMENTAL SECTION}

Chemicals and Standards. Acetonitrile, water, water with $0.1 \%$ formic acid (v/v), and formic acid (Optima, LC/MS grade) were purchased from Fisher Scientific (Leicestershire, U.K.), while ammonium formate (MS-grade) was supplied by Sigma-Aldrich (Steinheim, Germany). Standards of analytical purity for 639 metabolites and 14 internal standards (ISs) were obtained from various commercial sources, while 37 food metabolites were synthesized in-house, as detailed elsewhere (see Table S1 in the Supporting Information). ${ }^{18,19}$ Individual stock solutions for all 690 compounds were prepared at concentrations of $10000 \mathrm{mg} / \mathrm{L}$ for endogenous metabolites or $1000 \mathrm{mg} / \mathrm{L}$ for exogenous ones and ISs, using the solvent recommended by the supplier and/or literature, and stored at $-80{ }^{\circ} \mathrm{C}$. From these stock solutions, three multimetabolite working solutions were prepared at $50 \mathrm{mg} / \mathrm{L}$ in water:acetonitrile $(80: 20(\mathrm{v} / \mathrm{v}))$, and these were employed to build the calibration curves by serial dilution and to prepare spiked urine/plasma samples for validation purposes. We also prepared a fourth working solution by combining a set of 13 ISs, depicting the broad chemical diversity of the metabolite classes assayed (see Table S1). To optimize the extraction conditions and method validation, we employed anonymized pooled samples of urine and plasma collected in previous studies from healthy volunteers after 1 week of a lowpolyphenol diet. ${ }^{20}$

Sample Preparation. Urine samples were first centrifuged at $10000 \mathrm{~g}$ for $10 \mathrm{~min}$ at $4{ }^{\circ} \mathrm{C}$, and then the supernatants $(20$ $\mu \mathrm{L}$ ) were diluted 5-fold with $80 \mu \mathrm{L}$ of ultrapure water containing $0.1 \%$ formic acid (v/v) and $125 \mu \mathrm{g} / \mathrm{L}$ of the IS set previously described. Plasma samples $(100 \mu \mathrm{L})$ were first spiked with $10 \mu \mathrm{L}$ of $1 \mathrm{mg} / \mathrm{L}$ indoleacetic acid- $d_{2}$ in water (IS to monitor the extraction efficiency). Samples were subsequently mixed with $500 \mu \mathrm{L}$ of cold acetonitrile $\left(-20{ }^{\circ} \mathrm{C}\right)$ containing $1.5 \mathrm{M}$ formic acid and $10 \mathrm{mM}$ ammonium formate in a Sirocco Protein Precipitation plate (Waters, Milford, MA, USA), vortexed for $1 \mathrm{~min}$, and kept at $-20{ }^{\circ} \mathrm{C}$ for $10 \mathrm{~min}$ to promote protein precipitation (PPT). Then, a Waters Positive Pressure-96 Processor was employed to collect the extracts in 96-well collection plates, which were taken to dryness using a MaxiVac $\beta$ vacuum concentrator (Daejeon, South Korea). Finally, samples were reconstituted in $100 \mu \mathrm{L}$ of water:acetonitrile $(80: 20, \mathrm{v} / \mathrm{v})$ containing $0.1 \%$ formic acid $(\mathrm{v} / \mathrm{v})$ and $100 \mu \mathrm{g} / \mathrm{L}$ of the 13-IS set previously described and centrifuged at $1000 \mathrm{~g}$ for $5 \mathrm{~min}$; clean extracts then were transferred to 96-well plates for further analysis.

Multianalyte Targeted Metabolomics Analysis. Analyses were performed on a 1290 Infinity UHPLC system (Agilent, Santa Clara, CA, USA) coupled to a QTRAP 6500 mass spectrometer equipped with Ion Drive Turbo $\mathrm{V}$ ion source (Sciex, Framingham, MA, USA). Chromatographic separation was performed on a Luna Omega Polar C18 column, $100 \mathrm{~mm} \times 2.1 \mathrm{~mm}$ (i.d. $1.6 \mu \mathrm{m}$ ), equipped with a fully porous polar C18 security guard cartridge (Phenomenex, Torrance, CA, USA). Mobile phases employed under positiveion mode detection consisted of water (A) and acetonitrile (B), both containing $0.5 \%$ formic acid, by applying the following gradient program: $0-5 \mathrm{~min}, 5 \%-50 \% \mathrm{~B} ; 5-8 \mathrm{~min}$, $50 \%-100 \%$ B; $8-10 \mathrm{~min}, 100 \% \mathrm{~B} ; 10-10.1 \mathrm{~min}, 100 \%-5 \% \mathrm{~B}$; 10.1-12 $\mathrm{min}, 5 \% \mathrm{~B}$. In the negative-ion mode, water containing $0.1 \%$ formic acid and $10 \mathrm{mM}$ ammonium formate (A) and acetonitrile (B) were delivered as follows: 0-8 min, $5 \%-20 \%$ B; 8-10 min, 20\%-100\% B; 10-12 min, 100\% B; $12-12.1 \mathrm{~min}, 100 \%-5 \% \mathrm{~B} ; 12.1-14 \mathrm{~min}, 5 \% \mathrm{~B}$. Other chromatographic conditions were as follows: column temperature, $40{ }^{\circ} \mathrm{C}$; autosampler temperature, $4{ }^{\circ} \mathrm{C}$; injection volume, $2 \mu \mathrm{L}$; and flow rate, $0.5 \mathrm{~mL} / \mathrm{min}$. Mass spectrometry detection was performed under positive and negative ionization in separate runs, by using the scheduled multiple reaction monitoring (sMRM) mode. General MS parameters were as follows: ion spray voltage, $+4500 /-3500 \mathrm{~V}$; source temperature, $600{ }^{\circ} \mathrm{C}$; curtain gas, $30 \mathrm{psi}$; ion source gas 1 and gas 2 , 50 psi each; collision-activated dissociation gas, 3 psi; entrance potential, $\pm 10 \mathrm{~V}$; and target scan time, $0.05 \mathrm{~s}$. The MRM transitions were optimized by infusing standard solutions dissolved in mobile phase (proportion A:B 1:1 v/v, $500 \mu \mathrm{g} / \mathrm{L}$ ) into the mass spectrometer using a syringe pump at $5 \mu \mathrm{L} / \mathrm{min}$ flow rate (MSMS data are available in MetaboLights, study identifier: MTBLS1906). Complementarily, other metabolites for which authentic standards are not currently available were identified in real urine and plasma samples by means of product ion scan analysis $\left(\mathrm{MS}^{2}\right.$ and $\left.\mathrm{MS}^{3}\right)$ using the predicted nominal masses of these metabolites (level II identification according to the Metabolomics Standard Initiative), and their fragmentation conditions were experimentally optimized (see the subsection entitled "Method Optimization"). The semiquantification of these metabolites without standard was achieved by using the calibration curves of structurally similar metabolites, such as structural isomers (e.g., quantification of 2-methylbutyryl-L-carnitine with the calibration curve of valeryl-L-carnitine) or the corresponding aglycone for phase II metabolites (e.g., quantification of phloretin glucuronide with the calibration curve of phloretin). Optimized MRM 


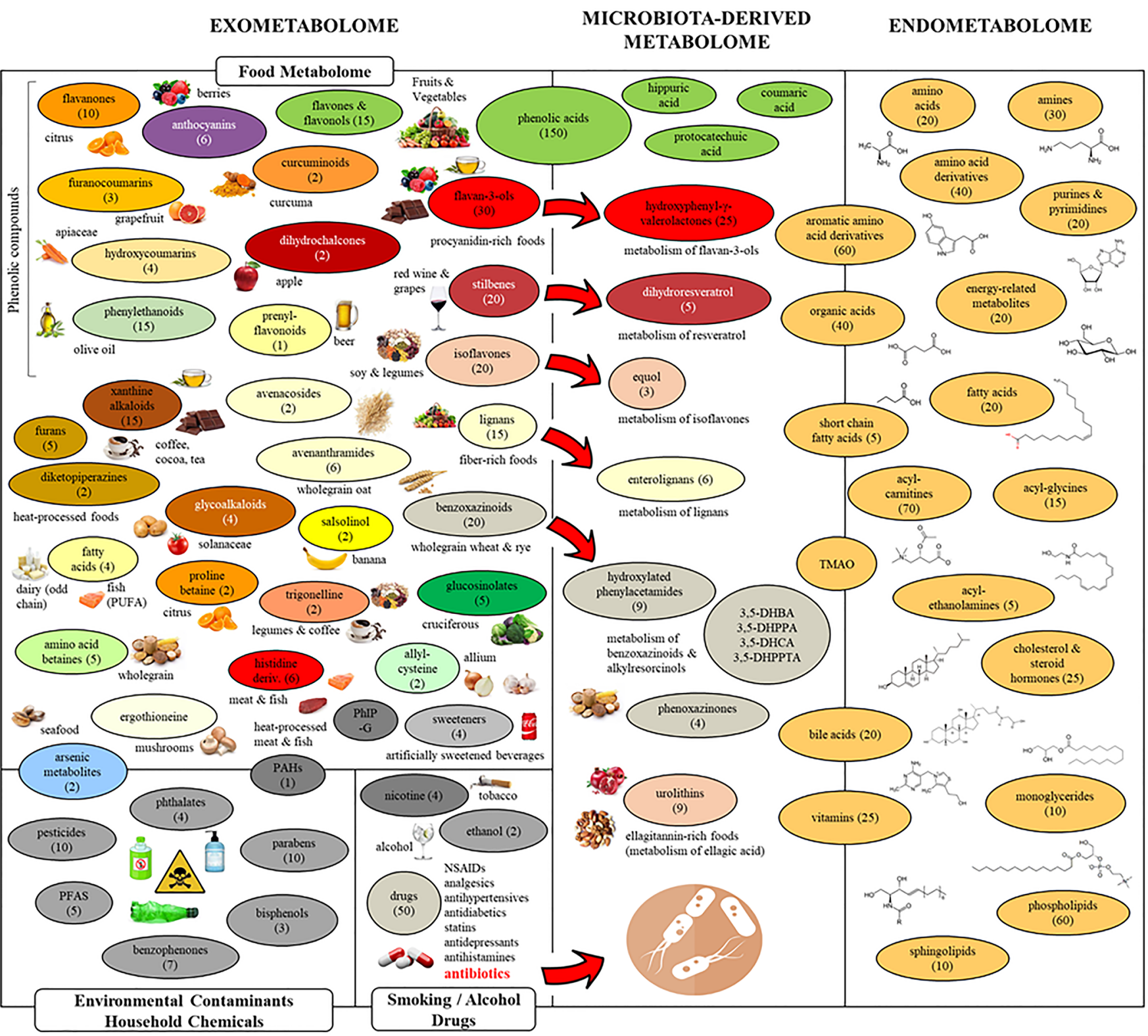

Figure 1. Graphical summary of the analytical coverage of the multimetabolite exposome-based metabolomics approach developed.

transitions, declustering potentials, collision energies, cell exit potentials, retention times and RT windows are listed in Table S2 in the Supporting Information. Analyst 1.6.2 and Sciex OSQ software (ABSciex, Framingham, MA, USA) were used for data acquisition and processing, respectively.

Analytical Validation. The optimized metabolomics approach was validated according to the requirements defined by the Center for Drug Evaluation and Research from the United States Food and Drug Administration (FDA) for bioanalytical method validation. ${ }^{21}$ The linearity was assessed by analyzing calibration curves, prepared in both solvent and blank urine/plasma, at 12 concentration levels within the range of $0.1-10000 \mu \mathrm{g} / \mathrm{L}$. The extraction recovery was determined by spiking blank urine/plasma samples at three concentration levels $(1,100,1000 \mu \mathrm{g} / \mathrm{L})$, which were analyzed in triplicate. Recovery percentages were calculated considering the endogenous levels found in blank samples using the following formula:

$$
\text { recovery }(\%)=\frac{\text { final concentration }- \text { initial concentration }}{\text { added concentration }} \times 100
$$

To assess matrix effects (ME), we compared the MS response of standards dissolved in solvent and extracted urine/plasma at the same concentration level $(1,100,1000 \mu \mathrm{g} / \mathrm{L})$. Method specificity was evaluated by testing the absence of interferences in blank solvents and extraction blanks, and by computing the retention time variability in solvent, blank urine/plasma and spiked urine/plasma along a 3-day analysis sequence. The limits of quantification (LOQ) were estimated in spiked urine/ plasma samples as the lowest concentration giving an average signal-to-noise $(\mathrm{S} / \mathrm{N})$ ratio of $>10$, measurable with an imprecision of $<20 \%$. These values were calculated by subtracting the analyte response observed in nonspiked samples. Intraday and interday precisions were evaluated by analyzing spiked urine/plasma samples at three concentration levels $(1,100,1000 \mu \mathrm{g} / \mathrm{L})$ five times within the same day, as well as on three consecutive days, respectively. Carryover was 
tested by analyzing blank water after injecting urine/plasma spiked at a concentration of $1000 \mu \mathrm{g} / \mathrm{L}$.

Application to a Dietary Intervention Study. For clinical validation of the metabolomics method optimized, we analyzed urine and plasma samples collected in a long-term dietary intervention. The study consisted of a controlled crossover trial with olive oil ( $80 \mathrm{~g} /$ day) lasting for one month in 10 healthy volunteers ( 6 males, 4 females, $40.4 \pm 4.1 \mathrm{y}$ ), following the general study design recommendations in exposure/nutritional assessment research. ${ }^{4}$ First-morning urine and fasting plasma samples were collected at baseline and at the end of the intervention period, and these were stored at $-80{ }^{\circ} \mathrm{C}$ until analysis. Urinary creatinine levels were analyzed using a Jaffe reaction-based kit (Siemens Healthineers, Erlangen, Germany) to normalize metabolomics data. The study was performed in accordance with the principles contained in the Declaration of Helsinki. The Bioethical Committee of the Hospital Virgen de la Victoria (Málaga, Spain) approved the study protocol, and all the participants provided written informed consent. The study was registered under ClinicalTrials.gov as NCT03101436.

Quality Control Assessment. Data quality control (QC) assessment was accomplished through a standardized protocol developed in-house, comprising the following steps: (i) data preprocessing, (ii) detection of sample outliers, (iii) sample quality assessment, and (iv) method reproducibility assessment. Data preprocessing included the removal of metabolites with more than $20 \%$ missing values in all the study groups and the imputation of the remaining missing values by using the KNN algorithm, ${ }^{22}$ and subsequent data normalization by applying log transformation and Pareto scaling. The distances to the group centroid then were computed based on Euclidean distances to detect outliers in the data matrix. After outlier removal, if needed, metabolites known to be influenced by preanalytical factors were employed to check the absence of abnormal values $( \pm 1.5 \times \mathrm{IQR})$, which could be indicative of improper handling/storage of blood (e.g., lactate) and/or urine (e.g., succinate) samples. ${ }^{23,24}$ Finally, reproducibility was evaluated by calculating the coefficients of variation for concentrations, retention times, and peak widths for ISs added to each sample.

Statistical Analysis. The metabolomics datasets obtained after analyzing urine and plasma samples from the clinical validation study were subjected to $t$-test statistical analysis to look for altered metabolites because of the intervention with olive oil. Fold changes were calculated using the following formula:

$$
\begin{aligned}
& \text { fold change }(\%) \\
& \qquad=\frac{\text { post-intervention concentration }- \text { basal concentration }}{\text { basal concentration }} \times 100
\end{aligned}
$$

\section{RESULTS}

Method Optimization. We compared various extraction methods for the comprehensive characterization of the human exposome, both in urine and plasma samples, considering more than 1000 metabolites, as illustrated in Figure 1. Simple dilution and reversed solid-phase extraction (SPE) (Oasis HLB) were tested for urine treatment, whereas plasma extraction was accomplished by means of protein precipitation (in tubes and in 96-well filtration plates), reversed solid-phase extraction (Oasis HLB), and hybrid protein precipitation with simultaneous SPE-mediated phospholipid removal (Ostro), as reported in our previously published studies focused on investigating the human food metabolome in urine ${ }^{18}$ and plasma $^{19}$ samples.

For urine, simple dilution provided the best performance in terms of coverage, enabling the simultaneous analysis of the wide range of metabolites assayed. To minimize matrix effects and obtain maximum sensitivity, 5-fold dilution was found to be the optimum procedure, since lower dilution factors provoked increased ion suppression, whereas minor metabolites became undetectable by applying higher dilutions. On the other hand, an in-plate PPT-based protocol was optimized for plasma analysis. The best extraction efficiency was obtained by using acetonitrile containing $1.5 \mathrm{M}$ formic acid and $10 \mathrm{mM}$ ammonium formate as the precipitation solvent, which allows interactions between proteins and metabolites to be reduced, especially for low-polarity ones (e.g., polyphenols, lipids). ${ }^{19}$ Noteworthy, similar extraction performance was observed using plasma volumes in the range of $20-100 \mu \mathrm{L}$, but with an inherent loss of sensitivity with lower volumes.

The chromatographic method was optimized to achieve maximum resolution and sensitivity for all the metabolites listed in Table S2, especially considering the presence of isomeric compounds, following a modification of the methodology described elsewhere. ${ }^{18,19}$ For this purpose, two different mobile phase sets and gradient elution programs were employed under positive and negative ionization modes to manage the chemical complexity of the human exposome. The use of formic acid and ammonium formate as mobile phase additives and an aprotic organic solvent (i.e., acetonitrile) in the negative ion mode provided satisfactory chromatographic resolution, reducing peak tailing for sulfate (e.g., sulfated steroids, phase II phenolics) and phosphate (e.g., organophosphate pesticides) metabolites, which consequently resulted in increased $\mathrm{S} / \mathrm{N}$ ratios and, therefore, improved analytical sensitivity. In contrast, chromatographic separation under positive polarity was performed by using more acidic mobile phases, which significantly enhanced electrospray ionization and provided better peak resolution for some specific metabolite classes (e.g., anthocyanins). ${ }^{25}$

For MS detection, the MRM transitions were optimized by infusing standard solutions into the mass spectrometer for 676 metabolites (+14 ISs). Furthermore, 352 additional metabolites were also included to expand the method coverage, considering other biologically important metabolites not commercially available (e.g., individual lipid species, exogenous phase II derivatives). To this end, urine/plasma samples were analyzed under the product ion scan $\left(\mathrm{MS}^{2}\right)$ mode by using predicted nominal masses of these metabolites, looking for expected fragments (e.g., $85 \mathrm{Da}$ for acylcarnitines, $184 \mathrm{Da}$ for lysophosphatidylcholines) or neutral losses (e.g., $176 \mathrm{Da}$ for glucuronides, $80 \mathrm{Da}$ for sulfates). If needed, $\mathrm{MS}^{3}$ fragmentation experiments were conducted to confirm the annotation. Compound-dependent parameters for these metabolites were experimentally optimized to achieve the highest sensitivity (see Table S2). Altogether, the metabolomics platform developed enables the simultaneous quantitation of 1019 metabolites in very short run times $(12 \mathrm{~min}+14 \mathrm{~min}$ in the positive- and negative-ion modes, respectively). Despite the huge number of transitions monitored, the minimum number of data points across chromatographic peaks was 15 , which ensures good accuracy and reproducibility. 

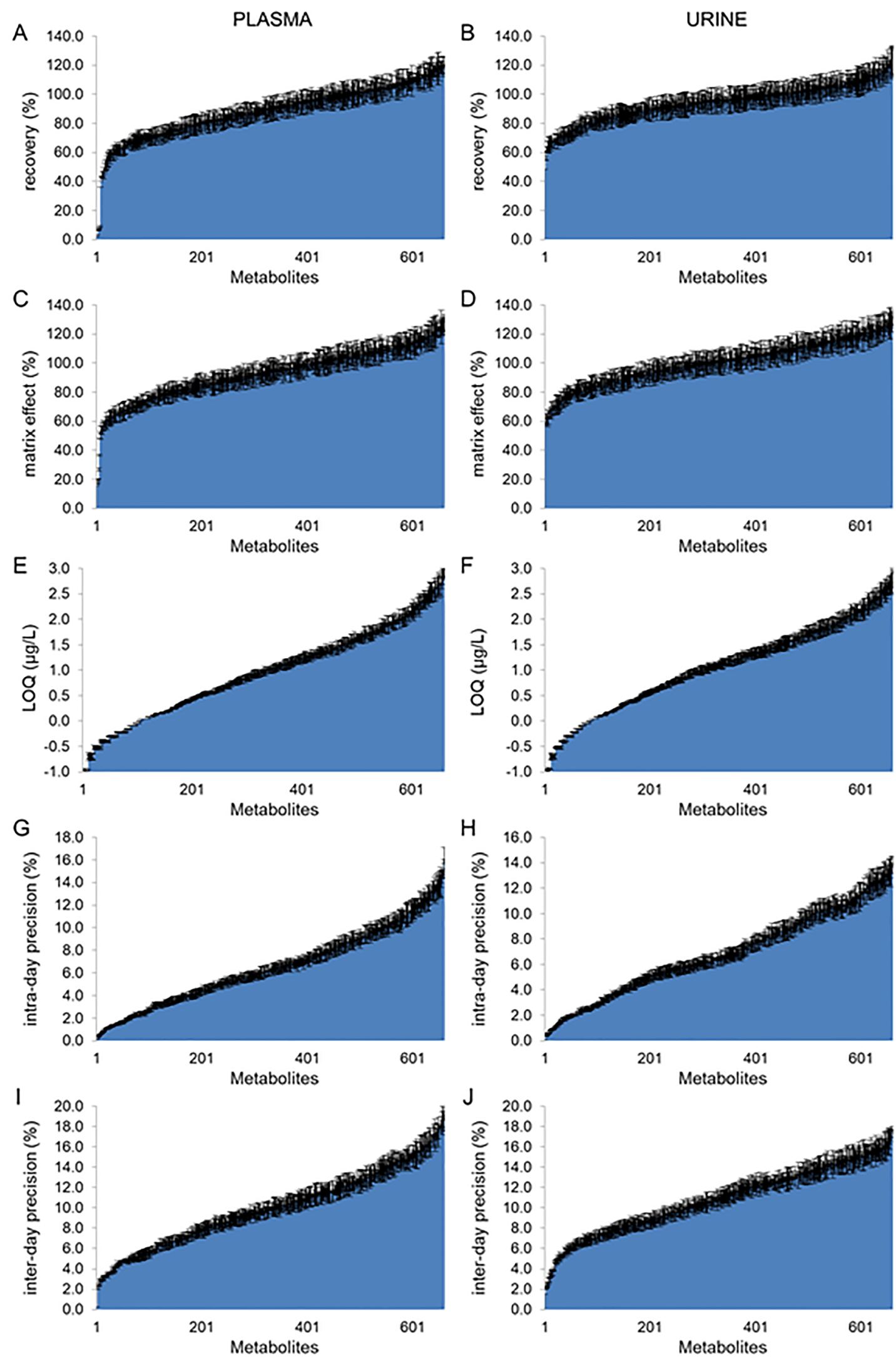

Figure 2. Column plots with standard deviation bars showing the distribution of recovery rates (for plasma (panel (A) and urine (panel (B)), matrix effects (for plasma (panel (C) and urine (panel (D)), logarithmic transformed limits of quantification (for plasma (panel (E) and urine (panel (F)), intraday precisions (for plasma (panel $(\mathrm{G})$ and urine (panel $(\mathrm{H})$ ), and interday precisions (for plasma (panel (I) and urine (panel (J)) for all the validated metabolites $(n=667)$ in plasma and urine. 
Analytical Validation. A conscientious analytical validation was performed according to the FDA guidelines, as summarized in Figure 2 (detailed information is given in Tables S3 and S4 in the Supporting Information). Calibration curves, prepared both in solvent and biological matrix, showed high linear responses over 3-5 orders of magnitude within the concentration range of $0.1-10000 \mu \mathrm{g} / \mathrm{L}$, with correlation coefficient $\left(R^{2}\right)$ values of $>0.99$ for all the metabolites. Satisfactory recovery rates $(70 \%-125 \%)$ were obtained for most of the validated metabolites $(85.6 \%$ and $95.8 \%$ of the total in plasma and urine, respectively) with negligible matrix effects (MEs) (see Figures S1 and S2 in the Supporting Information), which supports the possibility of using calibration curves prepared in solvent instead of matrixmatched calibrations to simplify the analytical workflow. Morepronounced ion suppression (ME 50\%-70\%) was observed for some polar metabolites detected in the void volume (e.g., polyamines) and flavonoid aglycones chromatographically coeluting with highly abundant phospholipids (only in plasma). Only for some sugar derivatives, the extent of ion suppression impeded their analysis in plasma samples (ME < $50 \%)$. Moreover, slightly lower recovery rates were found for the PPT-based extraction of some anthocyanins and isoflavones from plasma, which is consistent with previous findings. ${ }^{19}$ To assess the method specificity, the conventional procedure based on proving the lack of response in blank matrix was not suitable due to the endogenous presence of many of the metabolites assayed. As an alternative approach, we checked the absence of signal in blank solvents and extraction blanks, and we computed the retention time variability as a metric of the method's ability to differentiate a metabolite in the presence of potential interferences (i.e., closely coeluting structural isomers, e.g., leucine and isoleucine). Blank solvents and extraction blanks only showed appreciable levels of some fatty acids, phthalates, and bisphenol A, because of their ubiquitous presence in plastic ware (these blank levels should therefore be subtracted from concentrations found in real samples). On the other hand, retention time variability was $<3 \mathrm{~s}$ along a three-day analytical sequence for all the measured metabolites, both in solvent and biological matrix, thereby demonstrating the stability of the metabolomics platform and, consequently, its specificity to differentiate potential interferences. However, note that some isomeric metabolites were unresolved, because of the inherent limitations of reversed-phase chromatography (those marked with the symbol "§" in Table S2). The LOQs calculated for plasma and urine were very similar and indicated satisfactory sensitivity to detect most of the metabolites in real samples, according to the normal concentration ranges displayed in the Human Metabolome Database (HMDB) (IDs are listed in Table S2). These LOQs were lower than $50 \mu \mathrm{g} / \mathrm{L}$ for $\sim 75 \%$ of the metabolites monitored (Figure 2), with $10 \%$ of the metabolites being quantifiable at sub-ppb levels. Relative standard deviations for intraday and interday precision were $0.1 \%-16.4 \%$ and $0.3 \%-18.9 \%$ for plasma, whereas, for urine, they varied over the ranges of $0.1 \%-16.1 \%$ and $1.5 \%-19.6 \%$, respectively, below the $20 \%$ acceptance limit established by the FDA. $^{21}$ The analysis of blank solvent after injecting urine/ plasma samples spiked at $1000 \mu \mathrm{g} / \mathrm{L}$ evidenced appreciable carryover for some metabolite classes (e.g., acylcarnitines, pharmaceuticals). However, the signal detected in blanks was $<20 \%$ of the LOQ for these metabolites, within the acceptable range.
Application to a Dietary Intervention Study. The previously optimized and validated metabolomics platform was applied to urine and plasma samples collected from a dietary intervention study with olive oil to check its clinical potential. Most of the endogenous metabolites monitored (>90\%) were successfully quantified in $>80 \%$ of the samples analyzed, thereby demonstrating satisfactory method sensitivity. Among food-related metabolites, phenolic acids, methylxanthines, and other microbiota derivatives (e.g., enterolignans, hydroxyphenyl-valerolactones) were also regularly detected in urine and, to a lesser extent, in plasma samples. Similarly, other chemicals ubiquitously present in personal care products (e.g., parabens, phthalates, benzophenones) could also be found in most of the samples analyzed. However, the detection rate of other exogenous metabolites was much lower (e.g., flavonoids, drugs), thus indicating their specificity as exposure biomarkers.

These metabolomics data were first subjected to QC assessment to check the absence of analytical and preanalytical outliers, and to validate the analytical performance. No outlier was detected, and the coefficients of variation for the concentrations, retention times and peak widths for ISs were below $15 \%, 1 \%$, and $10 \%$, respectively, both in urine and plasma. Afterward, $t$-test analysis was applied to look for differential metabolites, because of the dietary intervention, as shown in Table 1.

\section{DISCUSSION}

This work presents the development of a novel multianalyte targeted metabolomics platform for large-scale quantitative exposome research, as illustrated in Figure 1. On the one hand,

Table 1. Differential Metabolites Identified in Urine and Plasma Samples $(t$-Test, $p<0.05)$ Following a 1-Month Intervention with Olive Oil

\begin{tabular}{|c|c|c|}
\hline metabolite & $p$-value & fold change (\%) \\
\hline \multicolumn{3}{|l|}{ Urine } \\
\hline hydroxytyrosol 4'-sulfate & 0.020787 & +1816.9 \\
\hline hydroxytyrosol 3 '-sulfate & 0.037823 & +1082.4 \\
\hline \multicolumn{3}{|l|}{ Plasma } \\
\hline ethanolamine & 0.001422 & +73.7 \\
\hline urea & 0.003088 & +16.1 \\
\hline s-adenosylmethionine & 0.003127 & +16.2 \\
\hline dimethylglycine & 0.005194 & +22.0 \\
\hline pyroglutamic acid & 0.010146 & +21.5 \\
\hline asymmetric dimethylarginine & 0.011142 & +46.5 \\
\hline trimethylamine & 0.011555 & +12.6 \\
\hline glutaryl-L-carnitine & 0.012186 & +27.3 \\
\hline succinic acid & 0.013465 & +16.9 \\
\hline azelaic acid & 0.014034 & +169.1 \\
\hline leucine & 0.014809 & +20.2 \\
\hline acetyl-L-carnitine & 0.016202 & +21.7 \\
\hline valine & 0.018121 & +20.3 \\
\hline s-adenosylhomocysteine & 0.018223 & +23.1 \\
\hline lysine & 0.018677 & +26.0 \\
\hline methionine & 0.019863 & +25.7 \\
\hline threitol & 0.026527 & +19.5 \\
\hline creatinine & 0.029948 & +19.9 \\
\hline glycochenodeoxycholic acid 3-glucuronide & 0.032348 & +178.2 \\
\hline indoleacetic acid & 0.036501 & +47.6 \\
\hline docosatetraenoic acid & 0.044264 & +6.8 \\
\hline phenylalanine & 0.047083 & +16.8 \\
\hline
\end{tabular}


we considered $\sim 500$ endogenous metabolites covering a broad range of chemical classes (e.g., amino acids and derivatives, organic acids, biogenic amines, vitamins, fatty acids, acylcarnitines, steroids). To build this endometabolome library, we focused on metabolites involved in central metabolic pathways according to metabolomics databases (e.g., HMDB, KEGG), also taking as a reference some metabolite kits commonly employed in metabolomics research (e.g., Biocrates MxP Quant 500 kit, IROA MSMLS) and related literature. ${ }^{26-28}$ Furthermore, 450 additional metabolites were also monitored to obtain a representative overview of the food metabolome and other lifestyle habits (e.g., smoking, alcohol consumption), based on the associations defined in the Food Biomarker Ontology. ${ }^{29}$ Besides these food-related metabolites, the method coverage also comprises other common xenobiotics, ${ }^{30}$ including pollutants (e.g., organophosphate pesticides, polycyclic aromatic hydrocarbons, arsenic metabolites), household chemicals (e.g., parabens, benzophenones, bisphenols, phthalates, perfluoroalkyl substances) and commonly consumed drugs (e.g., statins, analgesics, antibiotics, antidiabetics, antihypertensives, antiinflammatories, antidepressants, antihistamines), thereby enabling comprehensive exposure assessment. To investigate the role of microbiota in this complex interplay between external factors and endogenous processes, we also considered multiple microbiota-derived metabolites, such as biotransformed food components (e.g., urolithins, hydroxyphenyl-valerolactones), aromatic amino acid derivatives (e.g., neurotransmitters, indoles), short-chain fatty acids, bile acids, vitamins, and others.

The metabolomics platform developed thus enables the simultaneous detection of 1,019 metabolites in short run times (<30 min per sample). Furthermore, extraction methods optimized are simple, easily automatable (e.g., 96-well based robotic systems), and employ small sample volumes $(<20 \mu \mathrm{L}$ urine, $20-100 \mu \mathrm{L}$ plasma/serum). All this highlights the great applicability of the methodology in epidemiological research for dealing with large sample cohorts. Although achieving optimum analytical performance for all the metabolites under study at the same time is rather unrealistic, most of them were satisfactorily validated in terms of linearity, recovery, matrix effects, specificity, limits of quantification, intraday and interday precision, and carryover (Figure 2).

A few multimetabolite methods have previously been described for investigating specific metabolomics compartments, including the endometabolome, ${ }^{26-28}$ the food metabolome, ${ }^{18,19}$ the pharmaco-metabolome, ${ }^{31}$ and the toxicometabolome. ${ }^{32}$ However, to the best of our knowledge, the metabolomics platform optimized in this study represents the first approach to simultaneously characterize a larger swath of the exposome, providing much wider coverage in a single analysis. Furthermore, it is also noteworthy that our method consisted of multiple biotransformed exogenous metabolites (e.g., phase II derivatives), which allows the metabolism and detoxification processes of xenobiotics to be studied, unlike most of the conventional targeted metabolomics approaches based on enzymatic hydrolysis. This hydrolysis step is usually employed to reduce the metabolome complexity and simplify quantification, but it hinders the simultaneous analysis of multiple biomarkers, because optimal deconjugation conditions differ, depending on the metabolite class. ${ }^{33,34}$ This therefore emphasizes the great potential of the metabolomics approach developed here to obtain a comprehensive and realistic overview of the human exposome.

Finally, this methodology was successfully applied in an intervention trial with olive oil as a case study to demonstrate its applicability. Statistical analysis of the metabolomics data set showed a significant increase in urinary levels of hydroxytyrosol derivatives, validated biomarkers of olive oil intake. $^{29}$ On the other hand, the analysis of plasma samples revealed alterations in several metabolic pathways because of this intervention, including homeostasis of amino acids, onecarbon metabolism, and fatty acid oxidation (Table 1). This therefore demonstrates the complementarity of using both matrices in biomedical research, with exogenous metabolites being predominantly detected in urine, whereas blood samples provide more insights into endogenous processes and the phenotypic status.

\section{CONCLUSIONS}

In summary, this work describes, for the first time, the optimization, validation. and application of a multimetabolite platform for comprehensive and quantitative metabolomicsbased exposome research, with great utility in numerous research fields, because of the broad metabolome coverage, e.g., health research, nutrimetabolomics, toxicometabolomics, and pharmacometabolomics. The main strengths of this novel approach are (i) the ability to simultaneously quantify more than 1000 metabolites, (ii) both in urine and blood samples, (iii) in short-run times, (iv) by applying simple and automatable extraction methods, and (v) using small-sample volumes, which therefore facilitates its implementation in largescale epidemiological studies. On the other hand, the most important limitation of this methodology was the inherent inability of reversed-phase chromatography to resolve polar metabolites, which would make the development of orthogonal complementary approaches (e.g., hydrophilic interaction chromatography) necessary. Furthermore, the use of highthroughput extraction methods based on dilution and PPT, devised to allow for large-scale quantitation of as many metabolites as possible, might hinder the detection of some minor exogenous metabolites, which usually require specific extraction and cleanup procedures. To overcome this hurdle, solid-phase extraction procedures could be complementarily applied, as recently reported. ${ }^{18,19}$ Finally, note that standards were not available for many of the metabolites assayed. To partially solve this drawback, we included them in the multimetabolite MS method after being identified in real urine/plasma samples, thereby enabling their detection and semiquantification. We would like to make the point here that the method presented in this study is not intended to be definitive, but rather might undergo constant evolution and improvement with new metabolites.

This large-scale multianalyte targeted approach was tested in plasma and urine samples collected from a pilot dietary intervention trial with the aim of assessing the metabolomics coverage of this methodology in real samples, and exploring its ability to investigate diet-induced metabolomics alterations as a case study. In this regard, future studies are needed to assess the clinical potential of this metabolomics platform in larger sample cohorts. 


\section{ASSOCIATED CONTENT}

\section{(s) Supporting Information}

The Supporting Information is available free of charge at https://pubs.acs.org/doi/10.1021/acs.analchem.0c02008.

Extracted ion chromatograms for selected metabolites measured in solvent, spiked plasma and spiked urine for matrix effect assessment (PDF)

Tables S1-S4, listing commercial supplier for the metabolite standards, detailed MRM conditions, and validation parameters for plasma and urine (XLSX)

\section{AUTHOR INFORMATION}

\section{Corresponding Authors}

Cristina Andrés-Lacueva - Biomarkers and Nutrimetabolomics Laboratory, Department of Nutrition, Food Sciences and Gastronomy, Food Technology Reference Net (XaRTA), Nutrition and Food Safety Research Institute (INSA), and Faculty of Pharmacy and Food Sciences, University of Barcelona, 08028 Barcelona, Spain; CIBER Fragilidad y Envejecimiento Saludable (CIBERfes), Instituto de Salud Carlos III, Barcelona, Spain; 이이.org/0000-0002-8494-4978; Phone: +34 934034840; Email: candres@ub.edu; Fax: +34 934035931

Raúl González-Domínguez - Biomarkers and Nutrimetabolomics Laboratory, Department of Nutrition, Food Sciences and Gastronomy, Food Technology Reference Net (XaRTA), Nutrition and Food Safety Research Institute (INSA), and Faculty of Pharmacy and Food Sciences, University of Barcelona, 08028 Barcelona, Spain; CIBER Fragilidad y Envejecimiento Saludable (CIBERfes), Instituto de Salud Carlos III, Barcelona, Spain; ㅇo orcid.org/0000-00027640-8833; Phone: +34 934024513; Email: raul.gonzalez@ ub.edu; Fax: +34 934035931

\section{Authors}

Olga Jauregui - CIBER Fragilidad y Envejecimiento Saludable (CIBERfes), Instituto de Salud Carlos III, Barcelona, Spain; Scientific and Technological Center of University of Barcelona (CCiTUB), 08028 Barcelona, Spain

María Isabel Queipo-Ortuño - Unidad de Gestion Clínica Intercentros de Oncología Médica, Hospitales Universitarios Regional y Virgen de la Victoria, Instituto de Investigación Biomédica de Málaga (IBIMA)-CIMES-UMA, 29010 Málaga, Spain; CIBER Physiopathology of Obesity and Nutrition (CIBERobn), Instituto de Salud Carlos III, Madrid, Spain

Complete contact information is available at: https://pubs.acs.org/10.1021/acs.analchem.0c02008

\section{Notes}

The authors declare no competing financial interest.

\section{ACKNOWLEDGMENTS}

This work has received funding from the Spanish Ministry of Economy and Competitiveness (MINECO) (Nos. PCIN2015-229, PCIN-2015-238, and PCIN-2017-076) under the umbrella of the European Joint Programming Initiative "A Healthy Diet for a Healthy Life" (JPI HDHL, http://www. healthydietforhealthylife.eu), CIBERFES and ISCIII projects AC19/00111 and AC19/00096 (cofunded by the FEDER Program from EU, “A Way To Make Europe”), and from the Generalitat de Catalunya's Agency AGAUR (2017SGR1546). R.G.-D. thanks the "Juan de la Cierva" program from
MINECO (No. FJCI-2015-26590) and C.A.-L. acknowledges the ICREA Academia Award 2018. M.I.Q.-O. is the recipient of a "Miguel Servet Type II" fellowship (No. CPI13/00003 from ISCIII, cofunded by FEDER) and also belongs to the regional "Nicolas Monardes" research program (No. C-00302018, Junta de Andalucia, Spain). Authors thank to Prof. Paul Kroon (Quadram Institute Bioscience), Prof. Daniele Del Rio (University of Parma), and Prof. Kati Hanhineva (University of Eastern Finland) for kindly providing synthesized in-house standards.

\section{REFERENCES}

(1) Vermeulen, R.; Schymanski, E. L.; Barabási, A. L.; Miller, G. W. Science 2020, 367, 392-396.

(2) Wild, C. P. Cancer Epidemiol., Biomarkers Prev. 2005, 14, 18471850.

(3) Miller, G. W.; Jones, D. P. Toxicol. Sci. 2014, 137, 1-2.

(4) Ulaszewska, M. M.; Weinert, C. H.; Trimigno, A.; Portmann, R.; Andres Lacueva, C.; Badertscher, R.; Brennan, L.; Brunius, C.; Bub, A.; Capozzi, F.; et al. Mol. Nutr. Food Res. 2019, 63, No. e1800384.

(5) Griffin, J. L. Xenobiotica 2020, 50, 110-114.

(6) Ghanbari, R.; Sumner, S. Trends Mol. Med. 2018, 24, 197-205.

(7) Sakaguchi, C. A.; Nieman, D. C.; Signini, E. F.; Abreu, R. M.; Catai, A. M. Metabolites 2019, 9, 164.

(8) Nicholson, J. K.; Holmes, E.; Kinross, J.; Burcelin, R.; Gibson, G.; Jia, W.; Pettersson, S. Science 2012, 336, 1262-1267.

(9) Rowland, I.; Gibson, G.; Heinken, A.; Scott, K.; Swann, J.; Thiele, I.; Tuohy, K. Eur. J. Nutr. 2018, 57, 1-24.

(10) Rappaport, S. M.; Barupal, D. K.; Wishart, D.; Vineis, P.; Scalbert, A. Environ. Health Perspect. 2014, 122, 769-774.

(11) Dennis, K. K.; Marder, E.; Balshaw, D. M.; Cui, Y.; Lynes, M. A.; Patti, G. J.; Rappaport, S. M.; Shaughnessy, D. T.; Vrijheid, M.; Barr, D. B. Environ. Health Perspect. 2017, 125, 502-510.

(12) Bloszies, C. S.; Fiehn, O. Curr. Opin. Toxicol. 2018, 8, 87-92.

(13) Andra, S. S.; Austin, C.; Patel, D.; Dolios, G.; Awawda, M.; Arora, M. Environ. Int. 2017, 100, 32-61.

(14) Sobus, J. R.; Wambaugh, J. F.; Isaacs, K. K.; Williams, A. J.; McEachran, A. D.; Richard, A. M.; Grulke, C. M.; Ulrich, E. M.; Rager, J. E.; Strynar, M. J.; et al. J. Exposure Sci. Environ. Epidemiol. 2018, 28, 411-426.

(15) Brennan, L.; Hu, F. B. Mol. Nutr. Food Res. 2019, 63, No. e1701064.

(16) DiBattista, A.; Chakraborty, P. BMC Med. 2018, 16, 222.

(17) Ala-Korpela, M. Clin. Chem. 2018, 64, 30-33.

(18) González-Domínguez, R.; Urpi-Sarda, M.; Jáuregui, O.; Needs, P. W.; Kroon, P. A.; Andrés-Lacueva, C. J. Agric. Food Chem. 2020, 68, 1851-1861.

(19) González-Domínguez, R.; Jáuregui, O.; Mena, P.; Hanhineva, K.; Tinahones, F. J.; Angelino, D.; Andrés-Lacueva, C. Quantifying the human diet in the crosstalk between nutrition and health by multitargeted metabolomics of food and microbiota-derived metabolites. Int. J. Obes. 2020, DOI: 10.1038/s41366-020-0628-1.

(20) Rotches-Ribalta, M.; Urpi-Sarda, M.; Llorach, R.; BotoOrdoñez, M.; Jauregui, O.; Chiva-Blanch, G.; Perez-Garcia, L.; Jaeger, W.; Guillen, M.; Corella, D.; et al. J. Chromatogr. A 2012, $1265,105-113$.

(21) Center for Drug Evaluation and Research (CDER). Guidance for Industry: Bioanalytical Method Evaluation, 2001.

(22) Armitage, E. G.; Godzien, J.; Alonso-Herranz, V.; LópezGonzálvez, Á.; Barbas, C. Electrophoresis 2015, 36, 3050-3060.

(23) Kamlage, B.; Maldonado, S. G.; Bethan, B.; Peter, E.; Schmitz, O.; Liebenberg, V.; Schatz, P. Clin. Chem. 2014, 60, 399-412.

(24) Bernini, P.; Bertini, I.; Luchinat, C.; Nincheri, P.; Staderini, S.; Turano, P. J. Biomol. NMR 2011, 49, 231-243.

(25) Valls, J.; Millán, S.; Martí, M. P.; Borràs, E.; Arola, L. J. Chromatogr. A 2009, 1216, 7143-7172.

(26) Yan, Z.; Yan, R. Anal. Chim. Acta 2015, 894, 65-75. 
(27) Zhou, J.; Liu, H.; Liu, Y.; Liu, J.; Zhao, X.; Yin, Y. Anal. Chem. 2016, 88, 4478-4486.

(28) Li, K.; Naviaux, J. C.; Bright, A. T.; Wang, L.; Naviaux, R. K. Metabolomics 2017, 13, 122.

(29) Castellano-Escuder, P.; González-Domínguez, R.; Wishart, D. S.; Andrés-Lacueva, C.; Sánchez-Pla, A. Database 2020, 2020, No. baaa033.

(30) Center for Disease Control and Prevention (CDC). National Report on Human Exposure to Environmental Chemicals, 2017; available via the Internet at: https://www.cdc.gov/exposurereport/index.html, accessed March 2020.

(31) Qie, M.; Zhao, Y.; Yang, S.; Wang, W.; Xu, Z. J. Chromatogr. A 2019, 1608, 460423.

(32) Cortéjade, A.; Kiss, A.; Cren, C.; Vulliet, E.; Buleté, A. Talanta 2016, 146, 694-706.

(33) Quifer-Rada, P.; Martínez-Huélamo, M.; Lamuela-Raventos, R. M. Food Funct. 2017, 8, 2419-2424.

(34) Dwivedi, P.; Zhou, X.; Powell, T. G.; Calafat, A. M.; Ye, X. Chemosphere 2018, 199, 256-262. 